Sessions d'étude - Société canadienne d'histoire de l'Église catholique

\title{
La peinture murale dans les églises du Québec
}

\section{Laurier Lacroix}

Volume 47, 1980

URI : https://id.erudit.org/iderudit/1007110ar

DOI : https://doi.org/10.7202/1007110ar

Aller au sommaire du numéro

Éditeur(s)

Les Éditions Historia Ecclesiæ Catholicæ Canadensis Inc.

ISSN

0318-6172 (imprimé)

1927-7067 (numérique)

Découvrir la revue

Citer cet article

Lacroix, L. (1980). La peinture murale dans les églises du Québec. Sessions

d'étude - Société canadienne d'histoire de l'Église catholique, 47, 95-98.

https://doi.org/10.7202/1007110ar

Tous droits réservés @ Les Éditions Historia Ecclesiæ Catholicæ Canadensis Inc., 1980
Ce document est protégé par la loi sur le droit d'auteur. L'utilisation des services d'Érudit (y compris la reproduction) est assujettie à sa politique d'utilisation que vous pouvez consulter en ligne.

https://apropos.erudit.org/fr/usagers/politique-dutilisation/ 


\section{La peinture murale dans les églises du Québec *}

Par l'importance de la tradition des peintres muralistes et l'abondance de l'iconographie religieuse que les murales ont développée, le sujet de la peinture murale revêt un intérêt de tout premier ordre au Québec. Ce rapide exposé se propose de cerner ce qui apparaît dans l'état actuel de la recherche comme les points forts de son évolution. Il est important de se rappeler que la peinture murale pose des problèmes particuliers à l'artiste en ce qui a trait au sujet de l'œuvre (programme iconographique) et à sa composition qui doit être intégrée au bâtiment (localisation, éclairage).

Les tableaux de dévotion qui ornaient les églises et chapelles au Québec aux $\mathrm{XVII}^{\mathrm{e}}$ et $\mathrm{XVIII}^{\mathrm{e}}$ siècles n'avaient pas à répondre à ce caractère de permanence et d'unité avec le lieu dans lequel ils étaient placés. Appelée à vivre la vie même de l'édifice, la murale joue un rôle didactique plus complet que le tableau d'autel. En Nouvelle-France et au Bas-Canada, les tableaux ornant les églises étaient acquis au fil des circonstances, ils venaient s'ajouter à un bâtiment déjà existant ou remplacer une œuvre démodée ou en mauvais état. Alors que l'on assiste à un âge d'or de la sculpture sur bois dans le décor religieux au $\mathrm{XVIII}^{\mathrm{e}}$ siècle et au début du XIX $^{\bullet}$ (Levasseur, Baillargé, Quévillon), la venue de la collection Desjardins (1817 et 1820) semble marquer un renouveau d'intérêt pour la peinture dans le décor religieux.

Aucun artiste actif au Québec n'a utilisé la technique traditionnelle de la fresque ( buon fresco») mais plutôt celle de la «fresco secco» où le pigment est appliqué sur l'enduit du mur déjà sec. Le marouflage, qui consiste à peindre sur toile puis à encoller la toile sur le mur, deviendra prépondérant à la fin du $\mathrm{XIX}^{\mathrm{r}}$ siècle, des raisons économiques (l'artiste peut travailler dans son studio à un coût réduit) et pratiques (climat, matériaux accessibles) motivent l'adoption de ce procédé. L'intégration de la cire au pigment permet d'exécuter de grandes surfaces mates imitant le résultat obtenu avec la fresque.

* Résumé de la communication. 
Ce sont les artistes italiens et allemands actifs à Montréal et dans les régions du Québec qui apporteront la technique de la «fresco secco ». La qualité de leur métier, la nouveauté de leur style, l'engouement du clergé pour cette forme d'art européen au détriment des tableaux produits par les artistes locaux et la richesse de leur vocabulaire iconographique sont autant de raisons qui expiiquent ie succès obtenu par les fresquistes européens à partir de 1830. Angelo Pienovi, Daniel Muller. Julius Heldt, Luigi Cappelo, William Lamprecht sont parmi ces artistes qui ornent les nouveaux sanctuaires (Notre-Dame de Montréal, Gésù, Saint-Romuald) de leur important programme décoratif dont les longs cycles sont particulièrement adaptés aux dévotions pratiquées dans ces lieux.

\section{2) $1870-1890$}

Antoine Plamondon qui n'exécutera aucune murale et d'autres artistes canadiens protestèrent contre cette prise en main du marché et cette nouvelle mode de la décoration d'édifices par des artistes étrangers. Ce n'est qu'avec le retour d'Italie de Napoléon Bourassa que l'on voit des contrats accordés aux Canadiens. Bourassa, comme Muller au Gésù, pratique une esthétique nazaréenne qui préfère au sentiment dramatique et à la narrativité de l'iconographie de la ContreRéforme en vigueur jusqu'alors une imagerie plus symbolique comptant sur l'hiératisme des figures et l'utilisation d'attributs plus abstraits et intellectuels. Cette recherche de l'expression d'une spiritualité plus intérieure, première manifestation d'une redécouverte du Moyen-Âge, se manifeste dans la décoration de la chapelle Notre-Dame de Lourdes ou dans ses projets pour la décoration de la chapelle des Dominicains à Saint-Hyacinthe. Bourassa eut de nombreux émules parmi lesquels il faut mentionner l'excellent muraliste François-Xavier Meloche dont la décoration en grisaille pour Saint-Jean-Baptiste de Rouville (1886) par exemple, démontre sa maîtrise de l'espace et la richesse de son inspiration. Son cycle, comme dans le cas des meilleurs ensembles, présente une grande unité et une grande cohérence qui magnifie le lieu sans en transformer l'échelle.

\section{3) $1890-1930$}

Les réalisations antérieures à la période de 1890 seront suivies par un mouvement de grande expansion pour la peinture murale. Depuis 1870 déjà, des pays comme l'Angleterre, la France, l'Itąlie et maintenant les États-Unis reconnaissent l'intérêt d'orner les bâtiments publics de murales. Cette multiplication de commandes sur 
des chantiers de construction importants entraînera un essor dans l'expression et la recherche esthétique entourant la présentation de ces œuvres. Les places et les bâtiments publics s'ornent de cet art monumental et l'on veut élever le goût et la morale de la population par la propagation de sculptures et de tableaux. La peinture religieuse bénéficiera et encouragera largement la diffusion de ce nouveau vocabulaire pictural et l'on voit se généraliser les exemples d'églises ainsi décorées de murales. Cette période s'ouvre magistralement avec la décoration de la chapelle Notre-Dame du Sacré-Cœur à l'église Notre-Dame de Montréal à laquelle participent Joseph-C. Franchère, Ludger Larose, Joseph Saint-Charles, Henri Beau et Charles Gill. La Première messe à Ville-Marie et Le Serment de Dollard des Ormeaux intègrent des éléments d'histoire religieuse locale dans un cycle autrement consacré à des scènes de l'Ancien et du Nouveau Testament. Georges Delfosse (à qui l'on attribue la très belle réalisation de l'église du Très-Saint-Nom-de-Jésus de Montréal) continuera dans cette même veine à Saint-Jacques de Montréal. De nombreuses associations d'artistes se forment qui peuvent entreprendre de plus grands contrats et intégrer de façon plus systématique leurs compositions à l'ensemble architectural par les décorations des parties secondaires du bâtiment. On attache ainsi beaucoup d'importance aux bandes décoratives qui servent de lien visuel entre la composition généralement traitée de façon bi-dimensionnelle et le mur. Charles Huot, Napoléon Saint-Charles, Louis-E. Monty, C.-A. Chabauty, Joseph Saint-Hilaire, Préfontaine, Maxime Rousseau, Guido Nincheri seront alors très actifs comme muralistes. Il ne faut pas oublier d'inclure l'immense production réalisée par des religieuses qui décoraient principalement les chapelles de leurs institutions respectives.

Il nous apparaît cependant que c'est Ozias Leduc qui réussit le mieux à intégrer ses compositions à l'espace architectural et au milieu pour lequel ses œuvres sont destinées. Par l'utilisation d'une palette sensible épousant la couleur du lieu et par un choix de sujets appropriés il réussit, à Saint-Hilaire par exemple, mais encore à l'église du Saint-Enfant-Jésus-du-Mile-end de Montréal, à la chapelle épiscopale de Sherbrooke ou encore à Notre-Dame-de-la-Présentation de Shawinigan-sud, à diriger pleinement la haute spiritualité de son message vers le clergé et les fidèles.

\section{4) $1930-1960$}

Si l'on semble dédaigner la peinture murale dans le décor des églises et des centres communautaires que l'on construit de nos 
jours parce qu'elle est peu compatible avec la nouvelle liturgie et la multiplicité des fonctions auxquelles doivent servir ces locaux, la période qui a suivi la crise économique fut quand même la marque d'une nouvelle direction dans le développement de la peinture murale religieuse. Sous l'impulsion du renouveau de l'art sacré, en France particulièrement, les artistes actifs au Québec pratiquèrent un art mural axé sur le symbolisme s'exprimant par des formes très stylisées et abstraites misant sur les qualités expressives de la couleur et de la ligne. Profitant du séjour au Québec de figures intellectuelles et spirituelles importantes comme Dom Bellot et le dominicain AlainMarie Couturier, l'on voit des artistes comme André Lecoutey, Stanley Cosgrove. Wilfrid Corbeil, Marguerite Giguère Boileau, Simone Hudon, Lucien Martial exercer un art simplifié qui développe une iconographie insistant sur les principaux mystères de la foi.

Ce survol aura peut-être fait ressortir la richesse de ce mode d'expression et l'importance de ce patrimoine artistique religieux dont nous sommes les héritiers. Il importe que les personnes impliquées dans les comités d'art sacré prennent connaissance de la place du chaînon dont ils sont responsables dans ce grand mouvement de la peinture murale au Québec. La conservation et la restauration de ces œuvres doivent faire en sorte qu'elles continuent à livrer leur message dans le lieu pour lequel elles ont été conçues.

Laurier LACROIX, Histoire de l'art, Université Concordia. 